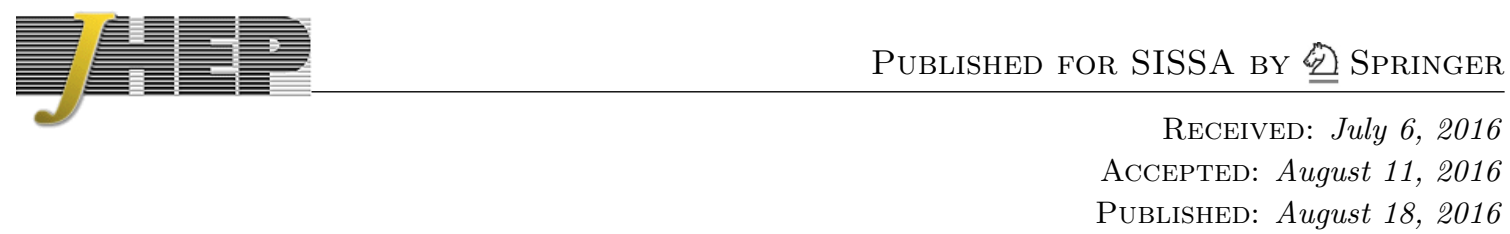

\title{
String moduli stabilization at the conifold
}

\author{
Ralph Blumenhagen, Daniela Herschmann and Florian Wolf \\ Max-Planck-Institut für Physik (Werner-Heisenberg-Institut), \\ Föhringer Ring 6, 80805 München, Germany \\ E-mail: blumenha@mpp.mpg.de, herschma@mpp.mpg.de, flowolf@mpp.mpg.de
}

ABSTRACT: We study moduli stabilization for type IIB orientifolds compactified on CalabiYau threefolds in the region close to conifold singularities in the complex structure moduli space. The form of the periods implies new phenomena like exponential mass hierarchies even in the regime of negligible warping. Integrating out the heavy conic complex structure modulus leads to an effective flux induced potential for the axio-dilaton and the remaining complex structure moduli containing exponentially suppressed terms that imitate nonperturbative effects. It is shown that this scenario can be naturally combined with the large volume scenario so that all moduli are dynamically stabilized in the dilute flux regime. As an application of this moduli stabilization scheme, a string inspired model of aligned inflation is designed that features a parametrically controlled hierarchy of mass scales.

KEYwords: Flux compactifications, Cosmology of Theories beyond the SM, Superstring Vacua

ARXIV EPRINT: 1605.06299 


\section{Contents}

1 Introduction 1

2 Type IIB fluxes on CY threefolds 3

2.1 Massless fields 3

2.2 Three-form flux 4

2.3 Large volume scenario 6

3 Moduli stabilization close to the conifold $\quad 7$

3.1 Periods of the quintic 7

3.2 Flux induced exponential mass hierarchies 9

$\begin{array}{lll}3.3 & \text { Conic LVS scenario } & 13\end{array}$

4 Towards axion alignment $\quad 15$

$\begin{array}{lll}4.1 & \text { Periods for } \mathbb{P}_{11226}[12] & 15\end{array}$

$\begin{array}{lll}4.2 & \text { Freezing axio-dilaton and complex structures } & 17\end{array}$

$\begin{array}{lll}4.3 & \text { Axion alignment } & 19\end{array}$

5 Conclusions $\quad 24$

\section{Introduction}

Moduli stabilization is one of the most important challenges to relate compactifications of string theory to our four-dimensional world. Despite its importance, we think it is fair so say that comparably few concrete scenarios have been discussed in the literature. The ingredients used are tree-level fluxes as well as perturbative and non-perturbative corrections to the leading order quantities.

The work of Giddings-Kachru-Polchinski (GKP) [1] revealed that Type IIB compactifications on warped Calabi-Yau threefolds equipped with localized sources and NS-NS and $\mathrm{R}-\mathrm{R}$ three-form fluxes are leading order solutions to the string equations of motion. There, a scalar potential for the complex structure and the axio-dilaton moduli is generated, while due to its no-scale structure, the Kähler moduli remain as massless moduli. It is from this scenario that the landscape idea of string vacua arose. In a second step, by turning on non-perturbative corrections to the superpotential, the no-scale structure was broken and the Kähler moduli could be stabilized. This led to the KKLT [2] and the large volume scenario (LVS) [3].

In [1] it was pointed out that one can dynamically freeze the complex structure moduli in the vicinity of a conifold singularity. For that purpose, as for the Klebanov-Strassler throat [4], one turns on a three-form flux on the three-cycle that vanishes at the conifold 
locus and an additional flux on its symplectic dual three-cycle. In this case, at the tip of the throat the warp factor becomes large, which leads to red-shifted masses of the modes localized there. From the statistical analysis [5] it even followed that the number of vacua enhances close to a conifold locus. This was explicitly verified for a concrete example in [6].

In this respect it is important to keep in mind that this analysis was employing the usual effective supergravity action described by the leading order Kähler potential and the Gukov-Vafa-Witten (GVW) superpotential [7, 8]. In the case of strong warping, this action is not any longer expected to be trustable as, due to red-shifting, certain KaluzaKlein modes can become light and one can have non-trivial mixings among the modes. The effective action for warped compactifications was studied in [9-12]. If one wants to use the standard supergravity action one has to ensure that one is working consistently in a dilute flux limit, where the backreaction is suppressed.

In this paper we systematically study such compactifications close to a conifold locus in the complex structure moduli space in the dilute flux limit. Our main concern is to analyze the appearing moduli mass scales and the implications for string cosmology model building. It is a known and celebrated result that, including a non-trivial warp factor, exponential hierarchies among the masses of the moduli are generated. This is true in particular for modes localized in a strongly warped throat. As a matter of fact, this is the idea of the Randall-Sundrum scenario [13]. Inflationary models in warped throats with red-shifted inflaton masses have recently been discussed in [14-16]. In this paper we find that even in the dilute flux limit, where the usual effective supergravity theory is applicable, exponential mass hierarchies between bulk modes are generated.

This observation is very interesting, as it allows to generate exponential hierarchies in a controllable supergravity theory, a problem that was recently of crucial relevance for potential realizations of large-field inflation in concrete string theory set-ups. To build models with large tensor-to-scalar ratios $r>0.01$ one needs a rolling of the inflaton over trans-Planckian field ranges, which are hard to control in a perturbation expansion (see e.g. [17] for a string realization). Here the perturbative shift symmetry of axions does help and various models of axion inflation were proposed (see e.g. [18-23] and [24] for review). However, it turned out to be quite a difficult task to dynamically stabilize all the moduli at a higher mass scale than the inflaton in a controllable way [25-27]. Moreover, such models of axion inflation came under pressure also via the weak-gravity conjecture (WGC) [28-31].

Having this motivation in mind, in this paper we will proceed as follows: in section 2 we briefly provide the main ingredients for Type IIB moduli stabilization, including geometric fluxes and instanton effects. For the quintic we recall the form of the periods close to the conifold singularity. The distinguished new issue is the appearance of a logarithmic term for a certain period. Computing the Kähler potential close to the conifold point, one realizes the appearance of axion-like shift-symmetries [32], making this regime interesting for realizing large field inflation. The latter has been subject of intense studies during the last two years, where it has become clear that the weak-gravity conjecture and concrete model building attempts severely constrain the viability of such models.

In section 3, we first study the no-scale scalar potential for the conic complex structure modulus and the axio-dilaton for the existence of minima close to the conifold locus. These 
indeed exist in a controllable way and for the mass of the conic complex structure modulus we find the two salient features:

- Independent of the overall size (Kähler modulus) of the Calabi-Yau, it is exponentially larger than the mass of the axio-dilaton.

- Requiring that its mass is lower than the string scale, leads to a size of the CY that implies the dilute flux limit, where warping can be neglected.

The second point is very satisfying, as it means that the usual supergravity action shows by itself its limitation to not strongly warped configurations. Integrating out the heavy conic complex structure modulus, one gets exponential terms in the axio-dilaton modulus that mimic non-perturbative effects. Second, we study whether the above scenario can be combined with the large volume scenario to dynamically provide an exponentially large overall volume of the CY.

section 4 is devoted to possible cosmological applications of this conic LVS scenario. For having more complex structure moduli available, we generalize the evaluation of the periods to the mirror of $\mathbb{P}_{11226}[12]^{(128,2)}$. Then the above mentioned exponentially suppressed, instanton like contributions to the effective action are investigated with respect to their potential to realize a string inspired model of aligned axion inflation. As in [25, 26, 33, 34], the inflaton is an axion-like complex structure modulus. We also critically reflect the pre-assumptions made for constructing this model and discuss the relation to a possible loop-hole in the mild version of the WGC.

\section{Type IIB fluxes on CY threefolds}

As in many previous works, we consider the issue of moduli stabilization for type IIB string theory compactified on orientifolds of Calabi-Yau threefolds $\mathcal{M}$ with $O 7$ and $O 3$-planes. Prior to turning on any fluxes, one obtains a moduli space parametrized by the vacuum expectation values of a set of scalar fields invariant under the orientifold projection.

\subsection{Massless fields}

The orientifold projection splits the cohomology groups into even and odd parts. The resulting massless closed string moduli fields [35] of the effective four-dimensional theory after compactification are summarized in table 1 . The axionic odd moduli do not play any role in the subsequent discussion so that we choose $h_{-}^{1,1}=0$. For convenience we also choose $h_{+}^{2,1}=0$ so that we do not get any additional abelian vector superfields.

The complex structure moduli $U^{i}$ are contained in the holomorphic three-form $\Omega_{3}$. The latter can be expanded in the basis of three-forms as follows

$$
\Omega_{3}=X^{\lambda} \alpha_{\lambda}-F_{\lambda} \beta^{\lambda} .
$$

where we denote a symplectic basis for the third cohomology of the Calabi-Yau manifold $\mathcal{M}$ by

$$
\left\{\alpha_{\Lambda}, \beta^{\Lambda}\right\} \in H^{3}(\mathcal{M}), \quad \Lambda=0, \ldots, h^{2,1},
$$




\begin{tabular}{|c|c|c|}
\hline number & modulus & name \\
\hline 1 & $S=e^{-\phi}-i C_{0}$ & axio-dilaton \\
$h_{-}^{2,1}$ & $U^{i}=v^{i}+i u^{i}$ & complex structure \\
$h_{+}^{1,1}$ & $T_{\alpha}=\tau_{\alpha}+i \rho_{\alpha}$ & Kähler \\
$h_{-}^{1,1}$ & $G^{a}=S b^{a}+i c^{a}$ & axionic odd \\
\hline
\end{tabular}

Table 1. Moduli in type IIB orientifold compactifications.

and $X^{\lambda}$ and $F_{\lambda}$ are the periods of the Calabi-Yau

$$
X^{\lambda}=\int_{A^{\lambda}} \Omega_{3}, \quad F_{\lambda}=\int_{B_{\lambda}} \Omega_{3} .
$$

Here $A^{\lambda}, B_{\lambda} \in H_{3}(\mathcal{M})$ denote a basis of Poincare dual three-cycles. The $X^{\lambda}$ can be considered as homogeneous coordinates of the complex structure moduli space. Inhomogeneous coordinates are e.g. defined via $U^{i}=X^{i} / X^{0}$ with $i=1, \ldots, h^{2,1}$.

In special geometry, the periods $F_{\lambda}$ can be expressed as derivatives $F_{\lambda}=\partial F / \partial X^{\lambda}$ of a prepotential $F$. Recall that in the large complex structure limit the prepotential takes the form

$$
F=\frac{d_{i j k} X^{i} X^{j} X^{k}}{X^{0}}, \quad i=1, \ldots, h^{2,1},
$$

where the constants $d_{i j k}$ are the triple intersection numbers of the mirror dual CY. At leading order in $\alpha^{\prime}$, the Kähler potential for these chiral superfields is given by

$$
K=-\log (S+\bar{S})-2 \log \mathcal{V}-\log \left(-i \int \Omega_{3} \wedge \bar{\Omega}_{3}\right)
$$

where $\mathcal{V}$ is the total volume of the CY expressed in terms of the four-cycle volumes $\operatorname{Re}\left(T_{\alpha}\right)$.

\subsection{Three-form flux}

For stabilizing the complex structure and the axio-dilaton moduli, we turn on type IIB three-form fluxes. The superpotential generating the corresponding F-term scalar potential is of the familiar Gukov-Vafa-Witten (GVW) type

$$
W=\int_{\mathcal{M}}(F+i S H) \wedge \Omega_{3}
$$

where $F=d C_{2}$ and $H=d B_{2}$ denote the R-R and NS-NS three-form field strengths. Since $W$ does not depend on the Kähler moduli, the scalar potential is of no-scale type

$$
V=e^{K}\left(G^{U \bar{U}} D_{U} W D_{\bar{U}} \bar{W}+G^{S \bar{S}} D_{S} W D_{\bar{S}} \bar{W}\right)
$$

with Minkowski minima at $F_{U}=D_{U} W=0$ and $F_{S}=D_{S} W=0$. The three-form fluxes also contribute to the D3-brane tadpole as $N_{\text {flux }}=\int H \wedge F$ so that for compensating the O3-plane tadpole one also needs localized sources in the form of D3-branes. 
It is well known that the backreaction of such a three-form flux and of localized D3branes on the geometry leads to a warped CY metric [1], i.e.

$$
d s^{2}=e^{2 A(y)} \eta_{\mu \nu} d x^{\mu} d x^{\nu}+e^{-2 A(y)} \tilde{g}_{m n} d y^{m} d y^{n}
$$

where the warp factor $A(y)$ only depends on the internal coordinates $y$ and $\tilde{g}_{m n}$ denotes the Ricci-flat metric on a CY threefold. Locally, the warp factor for a stack of D3-branes reads

$$
e^{-4 A(y)}=1+\frac{4 \pi g_{s} N}{|y|^{4}},
$$

i.e. it blows up close to the position of the D3-branes.

However, a warp factor is also induced by fluxes. Locally an $H_{3}$ form flux on an $A$ cycle and an $F_{3}$ form flux on its symplectic dual $B$-cycle leads to the warped metric on the deformed conifold. This can be described as a cone over $T^{1,1}$ cut off in the IR by a finite size $S^{3}$. Close to the tip, the warp factor is related to the value of the complex structure modulus $Z$ near the conifold [36] via $e^{A_{\text {con }}} \sim|Z|^{\frac{1}{3}}$. However, the moduli dependence is a bit more involved. Scaling the internal metric via $\tilde{g} \rightarrow \lambda^{2} \tilde{g}$ describes the breathing mode of the CY, i.e. the Kähler modulus for the overall volume. The relation is $\lambda \sim \mathcal{V}^{\frac{1}{6}}$. In [11] it was shown that the string equations of motion admit an unconstrained deformation $\lambda$ only if the warp factor scales non-trivially

$$
e^{-4 A(y)}=1+\frac{e^{-4 A_{\mathrm{con}}}}{\lambda^{4}} \sim 1+\frac{1}{\left(\mathcal{V}|Z|^{2}\right)^{\frac{2}{3}}} .
$$

Therefore, the warp factor can be approximated by a constant in the so-called dilute flux limit, which in this case takes the form

$$
\mathcal{V}|Z|^{2} \gg 1
$$

Note that in this limit the physical size of the three-cycle $A$

$$
\operatorname{Vol}(A)=\mathcal{V}^{\frac{1}{2}}\left|\int_{A} \Omega_{3}\right|=\left(\mathcal{V}|Z|^{2}\right)^{\frac{1}{2}}
$$

remains large, even with $|Z|$ becoming small.

Let us emphasize that, only in this limit, one can use the usual effective low-energy supergravity theory for the massless modes of the CY compactification. It has been argued that in the case of significant warping Kaluza-Klein modes localized in the throat are redshifted so that their mass is smaller than the mass of some of the stabilized former massless modes. Moreover, the derivation of a full effective theory for the strongly warped case has turned out to be a tough exercise [9-12] with additional subtleties arising from mixing of the modes and the necessity to introduce compensator fields.

Throughout this paper we will work in the dilute flux limit and will investigate to what extent one can achieve moduli stabilization close to the conifold singularity, i.e. for small $Z$. Special emphasis is dedicated on the question whether new features arise that are not present for the stabilization of the complex structure moduli in the large complex structure regime. 


\subsection{Large volume scenario}

Up to now the Kähler moduli remained as flat directions so that one could simply choose the total volume of the Calabi-Yau sufficiently large so that one stays in the dilute flux limit. Since the dilute flux limit requires that we fix the total volume $\mathcal{V}$ at exponentially large values, the natural way seems to combine the previous framework with the large volume scenario (LVS).

Let us briefly recall the main aspects of the LVS. There, one considers a swiss-cheese Calabi-Yau threefold with the $\alpha^{\prime}$-corrected Kähler potential

$$
K=-2 \log \left(\tau_{b}^{\frac{3}{2}}-\tau_{s}^{\frac{3}{2}}+\frac{\xi}{2} \operatorname{Re}(S)^{\frac{3}{2}}\right),
$$

with $\xi=-\frac{\chi(M) \zeta(3)}{2(2 \pi)^{3}}$ and where for simplicity we are choosing a CY manifold with only two Kähler moduli. In addition one has the GVW superpotential (2.6) corrected by a non-perturbative effect like an euclidean D3-brane instanton or a gaugino condensate on a stack of D7-branes

$$
W_{\mathrm{LVS}}(T)=W_{0}+A_{s} e^{-a_{s} T_{s}} .
$$

Here $W_{0}$ is the value of the GVW-superpotential, after integrating out the axio-dilaton and the complex structure moduli. For the LVS minimum to exist one needs $\chi(M)<0$, i.e. $h^{2,1}(M)>h^{1,1}(M)$. Up to CY-geometry dependent coefficients of order one, after freezing the axion $\rho_{s}$, the relevant terms in the scalar potential read

$$
V_{\mathrm{LVS}}(T)=e^{K_{c s}} \frac{g_{s}}{2}\left(\frac{\left|a_{s} A_{s}\right|^{2} \sqrt{\tau_{s}} e^{-2 a_{s} \tau_{s}}}{\mathcal{V}}-\frac{W_{0}\left|a_{s} A_{s}\right| \tau_{s} e^{-a_{s} \tau_{s}}}{\mathcal{V}^{2}}+\frac{\xi W_{0}^{2}}{g_{s}^{\frac{3}{2}} \mathcal{V}^{3}}\right) .
$$

Here $K_{c s}$ denotes the Kähler potential for the complex structure moduli. In the nonsupersymmetric AdS-type large-volume minimum the Kähler moduli get stabilized at

$$
\tau_{s}=\frac{(4 \xi)^{\frac{2}{3}}}{g_{s}}, \quad \mathcal{V}=\frac{W_{0} \xi^{\frac{1}{3}}}{2^{\frac{1}{3}} g_{s}^{\frac{1}{2}}\left|a_{s} A_{s}\right|} e^{a_{s} \tau_{s}}
$$

Note that the scalar potential $V_{\text {LVS }}$ close to the LVS-minimum is $1 / \mathcal{V}$ suppressed relative to the former no-scale potential for the complex structure and the axio-dilaton moduli.

The canonically normalized Kähler moduli masses can be computed as the eigenvalues of the matrix $\left(M^{2}\right)^{i}{ }_{j}=\frac{1}{2} K^{i k} \partial_{k} \partial_{j} V$, where the inverse of the Kähler metric is given at leading order in $1 / \mathcal{V}$ as

$$
\begin{aligned}
K^{\tau_{b} \bar{\tau}_{b}} & =\frac{4}{3} \mathcal{V}^{\frac{4}{3}}, \quad K^{\tau_{s} \bar{\tau}_{s}}=\frac{8}{3} \sqrt{\tau_{s}} \mathcal{V} \\
K^{\tau_{b} \bar{\tau}_{s}} & =K^{\tau_{s} \bar{\tau}_{b}}=4 \tau_{s} \mathcal{V}^{\frac{2}{3}}
\end{aligned}
$$

At leading order, the masses of the Kähler moduli are

$$
\begin{aligned}
& M_{\tau_{b}}^{2} \sim O(1) \frac{W_{0}^{2} \xi}{g_{s}^{\frac{1}{2}} \mathcal{V}^{3}} M_{\mathrm{pl}}^{2}, \quad M_{\rho_{b}}^{2} \sim 0, \\
& M_{\tau_{s}}^{2} \sim M_{\rho_{s}}^{2} \sim O(1) \frac{a_{s}^{2} W_{0}^{2} \xi^{\frac{4}{3}}}{g_{s} \mathcal{V}^{2}} M_{\mathrm{pl}}^{2} .
\end{aligned}
$$

For later purposes, note that the masses do not depend on the parameter $A_{s}$. 


\section{Moduli stabilization close to the conifold}

In this section we first provide the form of the periods for the quintic in the vicinity of the conifold singularity. This serves as the prototype example for the subsequent analysis of dynamical moduli stabilization. In our analysis we restrict ourselves to the (final) dynamical moduli: axio-dilaton, the complex structure governing the size of the three-cycle vanishing at the conifold locus and the Kähler moduli.

\subsection{Periods of the quintic}

As a concrete example of a Calabi-Yau manifold featuring a conifold singularity, we consider the mirror dual of the quintic $\mathbb{P}_{4}[5]^{(101,1)}$, whose single complex structure modulus is given by the complex parameter $\psi$ in the hypersurface constraint

$$
P=\sum_{i=1}^{5} Z_{i}^{5}-5 \psi \prod_{i=1}^{5} Z_{i}=0 .
$$

For the co-dimension one locus $\psi=1$, this hypersurfaces becomes singular, i.e. $P=\partial_{i} P=0$ for $i=1, \ldots, 5$. The second derivatives do not vanish so that one has a conifold singularity. It is known that at the singularity $u=5(\psi-1)=0$, a three-cycle $B^{1}$ shrinks to zero size, i.e. the corresponding period has to vanish like $F_{1}=\int_{B^{1}} \Omega_{3} \sim u+O\left(u^{2}\right)$. Moreover, for a closed loop around the conifold singularity, the symplectic dual period undergoes a monodromy $X^{1} \rightarrow X^{1}+F_{1}$. The remaining periods should stay finite at the conifold locus.

For the concrete model of the mirror of the quintic, in the regime $|\psi|<1$ a basis of periods solving the Picard-Fuchs equation can be derived from the fundamental period [37]

$$
\varpi_{f}(\psi)=-\frac{1}{5} \sum_{n=1}^{\infty} \frac{\lambda^{2 n} \Gamma\left(\frac{n}{5}\right)(5 \psi)^{n}}{\Gamma(n) \Gamma^{4}\left(1-\frac{n}{5}\right)},
$$

via

$$
\varpi_{i}(\psi)=-\left(\frac{2 \pi i}{5}\right)^{3} \varpi_{f}\left(\lambda^{i} \psi\right)
$$

with $i=0,1,2,4$ and $\lambda=\exp (2 \pi i / 5)$. These do not yet form a symplectic basis, which was determined explicitly in [37]. When expanded around the conifold locus $u \sim 0$ they take the form $[38,39]$

$$
\begin{aligned}
F_{0} & =\tilde{a}_{0}+\tilde{b}_{0} u+\ldots, \\
F_{1} & =a u+\ldots \\
X^{0} & =a^{0}+b^{0} u+\ldots, \\
X^{1} & =-\frac{1}{2 \pi i} F_{1} \log u+c+d u+\ldots
\end{aligned}
$$


with the following numerical values of the parameters

$$
\begin{aligned}
a & =\frac{\sqrt{5}}{2 \pi i}, \quad c=1.07072586843016, \quad d=-0.0247076138044847 \\
a^{0} & =1.29357398450411 i, \quad b^{0}=0.15076669512354743 i \\
\tilde{a}_{0} & =6.19501627714957-7.114656914772605 i \\
\tilde{b}_{0} & =1.016604716702582-0.8292168231795108 i .
\end{aligned}
$$

Half of the periods can be considered as homogeneous coordinates on the complex structure moduli space. Now we introduce inhomogeneous coordinates by dividing by the period $X^{0}$. Introducing the new complex structure modulus

$$
Z=\frac{F_{1}}{X^{0}}=\frac{a}{a^{0}} u+O\left(u^{2}\right),
$$

which in the following we also call the "conic" modulus, the period vector $\Pi^{T}=$ $\left(F_{0}, F_{1}, X^{0}, X^{1}\right)$ can be expressed as

$$
\Pi=X^{0}\left(\begin{array}{c}
\tilde{A}_{0}+\tilde{B}_{0} Z+O\left(Z^{2}\right) \\
Z \\
1 \\
-\frac{1}{2 \pi i} Z \log Z+C+D Z+O\left(Z^{2}\right)
\end{array}\right)
$$

with parameters

$$
\begin{aligned}
\tilde{A}_{0} & =\frac{\tilde{a}_{0}}{a^{0}}=-\frac{11}{2}-4.7906993443009 i \\
\tilde{B}_{0} & =\frac{a^{0} \tilde{b}_{0}-b^{0} \tilde{a}_{0}}{a a^{0}}=-0.82772681057019 i \\
C & =\frac{c}{a^{0}}=-0.82772681057019 i=\tilde{B}_{0} \\
D & =\frac{1}{a}\left(d-\frac{b^{0} c}{a^{0}}+\frac{a}{2 \pi i} \log \left(\frac{a}{a^{0}}\right)\right)=-0.2146873099644409 i
\end{aligned}
$$

Note the non-trivial relation $C=\tilde{B}_{0}$. For these values, the corresponding Kähler potential for the complex structure modulus is given by

$$
\begin{aligned}
K_{\mathrm{cs}} & =-\log \left[-i \Pi^{\dagger} \Sigma \Pi\right] \\
& =-\log \left[\frac{1}{2 \pi}|Z|^{2} \log \left(|Z|^{2}\right)+A+O\left(|Z|^{2}\right)\right]
\end{aligned}
$$

with $A=9.57814$ and the symplectic pairing

$$
\Sigma=\left(\begin{array}{cccc}
0 & 0 & 1 & 0 \\
0 & 0 & 0 & 1 \\
-1 & 0 & 0 & 0 \\
0 & -1 & 0 & 0
\end{array}\right)
$$


Note that the linear terms in $K_{\mathrm{cs}}$ cancel so that, as advocated in [32], the Kähler potential respects the continuous shift symmetry $Z \rightarrow e^{i \theta} Z$. In section 4.1 we generalize these results even up to second order to the mirror of the Calabi-Yau threefold $\mathbb{P}_{11226}[12]^{(128,2)}$ that features two complex structure moduli.

Throughout this paper we consider these two Calabi-Yaus as prime examples of complex structure moduli spaces developing a conifold singularity. However, when analyzing concrete models of moduli stabilization, we allow ourselves some more flexibility with respect to the concrete values of the numerical parameters in the periods.

\subsection{Flux induced exponential mass hierarchies}

Let us now discuss whether the GVW-superpotential admits vacua in which the complex structure (conic) modulus $Z$ is dynamically stabilized close to the conifold singularity $Z=0$. The first concrete proposal of this has been made in [1], which we also take as our starting point.

Stabilizing the conic modulus. Consider a CY like the mirror of the quintic that develops a conifold singularity at $Z=0$ and which has four periods that around the conifold locus $Z \sim 0$ admit an expansion (3.7)

$$
\begin{aligned}
X^{0} & =1, & X^{1} & =-\frac{1}{2 \pi i} Z \log Z+\tilde{B}_{0}+D Z+\ldots \\
F_{0} & =\tilde{A}_{0}+\tilde{B}_{0} Z+\ldots, & F_{1} & =Z .
\end{aligned}
$$

Now we freeze the complex structure modulus $Z$ by turning on three-form fluxes so that the superpotential reads

$$
\begin{aligned}
W & =f X^{1}+i h S F_{1}-i h^{\prime} S X^{0} \\
& =f\left(-\frac{1}{2 \pi i} Z \log Z+\tilde{B}_{0}+D Z+\ldots\right)+i h S Z-i h^{\prime} S .
\end{aligned}
$$

Let us first freeze the complex structure modulus via $F_{Z}=0$, which up to terms that vanish in the $Z \rightarrow 0$ limit leads to

$$
\underbrace{\frac{f}{2 \pi i} \log Z-i h S}_{\text {order } \log Z} \underbrace{+\frac{f}{2 \pi i}-D f}_{\text {order } O(1)}+\ldots=0 .
$$

Note that this contribution entirely comes from the $\partial_{Z} W$ term in $F_{Z}$ and that the contribution from $\left(\partial_{Z} K\right) W$ in $F_{Z}$ is subleading. ${ }^{1}$ Moreover, we assume that, after all, the axio-dilaton can be stabilized such that $h /\left(f g_{s}\right) \gg 1$ and that $h \gg h^{\prime}$. Therefore at leading order one finds

$$
Z \sim \hat{C} e^{-\frac{2 \pi h}{f} S}, \quad \text { with } \quad \hat{C}=\exp (-1+2 \pi i D)
$$

\footnotetext{
${ }^{1}$ While the present work was almost finished (see [40]), this order one correction in (3.13) has also been observed in [34].
} 
so that for a sufficiently large exponent one can indeed stabilize the complex structure modulus close to the conifold singularity. ${ }^{2}$

After invoking the relation (3.13), the second $F$-term $F_{S}$ yields

$$
D_{S} W=\left(i h \hat{C} e^{-\frac{2 \pi h}{f} S}-i h^{\prime}\right)-\frac{1}{S+\bar{S}}\left(\tilde{B}_{0} f+\frac{f}{2 \pi i} \hat{C} e^{-\frac{2 \pi h}{f} S}-i h^{\prime} S\right) .
$$

We observe that this F-term is identical to the one that can be derived by inserting the solution (3.14) directly into the superpotential (3.12) to obtain an effective superpotential for the axio-dilaton modulus

$$
W_{\text {eff }}=\tilde{B}_{0} f+\frac{f}{2 \pi i} \hat{C} e^{-\frac{2 \pi h}{f} S}-i h^{\prime} S+\ldots
$$

This superpotential features the two striking features

- Besides flux induced polynomial terms like $i h^{\prime} S$ the effective superpotential contains also infinitely many exponentially suppressed terms that, though also flux induced, have the same form as coming from some $D(-1)$ instantons.

- The exponential term suggests that the continuous shift symmetry $S \rightarrow S+i \theta$ is broken to a discrete one by the non-vanishing $h$-flux. This is evident from the superpotential (3.12), as a discrete shift of the universal axion can be compensated by changing the branch of the $\log Z$-term in $W$.

Therefore, the effective superpotential (3.16) offers the possibility to mimic the behavior of non-perturbative effects via flux induced tree-level contributions to $W$. Before we employ (3.16) to stabilize the axio-dilaton such that $h /\left(f g_{s}\right) \gg 1$, let us compute the mass of the conic modulus $Z$.

Mass of conic modulus. Since the complex structure modulus $Z$ is fixed via $D_{Z} W=0$, for determining its mass we can evaluate

$$
V_{Z \bar{Z}}=\partial_{Z} \partial_{\bar{Z}} V=\left.e^{K} G^{Z \bar{Z}} \partial_{Z}\left(D_{Z} W\right) \partial_{\bar{Z}}\left(D_{\bar{Z}} \bar{W}\right)\right|_{D_{Z} W=0} .
$$

In our case $D_{Z} W$ is holomorphic at leading order and hence the masses of the two real scalars in $Z$ are degenerate. For the metric one obtains at leading order

$$
G_{Z \bar{Z}} \sim-\frac{1}{2 \pi A} \log \left(|Z|^{2}\right)
$$

so that

$$
V_{Z \bar{Z}} \sim-\frac{1}{2 \operatorname{Re}(\mathrm{S}) \mathcal{V}^{2}|Z|^{2}} \frac{f^{2}}{2 \pi \log \left(|Z|^{2}\right)}
$$

With $M_{Z}^{2}=\frac{1}{2} G^{Z \bar{Z}} V_{Z \bar{Z}}$, the mass of the canonically normalized complex structure modulus becomes

$$
M_{Z}^{2} \sim \frac{M_{\mathrm{pl}}^{2}}{4 \operatorname{Re}(\mathrm{S}) \mathcal{V}^{2}|Z|^{2}} \frac{A f^{2}}{\log ^{2}\left(|Z|^{2}\right)}
$$

\footnotetext{
${ }^{2}$ For the quintic one finds $\hat{C}=\exp (0.35)$ so that $\hat{C} \in \mathbb{R}$.
} 
Using the expression for the string scale, $M_{\mathrm{s}}^{2}=\frac{M_{\mathrm{pl}}^{2} g_{s}^{\frac{1}{2}}}{\mathcal{V}}$, one can write

$$
M_{Z}^{2} \sim \frac{M_{\mathrm{s}}^{2}}{\mathcal{V}|Z|^{2}} \frac{A f^{4} g_{s}^{\frac{5}{2}}}{16 \pi^{2} h^{2}} .
$$

Due to the $|Z|^{2} \sim \exp \left(-\frac{4 \pi h}{f g_{s}}\right)$ factor in the denominator this mass is exponentially enhanced so that it only makes sense for $\mathcal{V}|Z|^{2} \gg 1$, i.e. for exponentially large volume. To summarize:

- The mass of the conic modulus $Z$ comes out exponentially enlarged so that the volume eventually has to be chosen/frozen at exponentially large values. This makes the large volume scenario the natural candidate for Kähler-moduli stabilization.

- The used effective supergravity theory by itself indicates its limitation, i.e. that it is applicable only in the dilute flux regime $\mathcal{V}|Z|^{2} \gg 1$ where warping is negligible.

Mass of axio-dilaton. For stabilizing the axio-dilaton and computing its mass, let us distinguish the two cases whether the flux $h^{\prime}$ vanishes or not.

Case $A: h^{\prime} \neq 0$

In this case the exponential term in the effective superpotential (3.16) can be neglected against the linear term so that

$$
W_{\text {eff }}=\tilde{B}_{0} f-i h^{\prime} S
$$

and

$$
D_{S} W_{\mathrm{eff}}=-\frac{1}{S+\bar{S}}\left(i h^{\prime} \bar{S}+\tilde{B}_{0} f\right)
$$

We assume $i \tilde{B}_{0} \in \mathbb{R}$ so that one gets the stabilized axio-dilaton

$$
\frac{1}{g_{s}}=i \tilde{B}_{0} \frac{f}{h^{\prime}}, \quad C_{0}=0 .
$$

For the quintic one finds $i \tilde{B}_{0} \sim 0.83$ so that we should choose $h^{\prime}>0$. For the complex structure modulus we thus obtain

$$
Z \sim \hat{C} \exp \left(-2 \pi i \tilde{B}_{0} \frac{h}{h^{\prime}}\right)
$$

For fixing $g_{s}<1$ in the perturbative regime and to have $Z$ close to the conifold we require $h>h^{\prime}>0$ and $f>h^{\prime}>0$. Note that there is no restriction on the the relative size of $h$ and $f$.

The degenerate mass of the complex axio-dilaton can be determined straightforwardly as

$$
M_{S}^{2}=\frac{M_{\mathrm{pl}}^{2}}{\mathcal{V}^{2} \operatorname{Re}(S)} \frac{\left|\tilde{B}_{0} f\right|^{2}}{A}
$$


so that one obtains

$$
\frac{M_{S}^{2}}{M_{Z}^{2}} \sim|Z|^{2} \sim \exp \left(-4 \pi i \tilde{B}_{0} \frac{h}{h^{\prime}}\right),
$$

i.e. the mass of the axio-dilaton is exponentially suppressed against the mass of the complex structure modulus. Note that this a posteriori justifies the use of the effective superpotential (3.16) for the axio-dilaton modulus. Let us emphasize that this effect is not due to warping, as it remains in the dilute flux limit. In the strongly warped case it is usually the red-shifted modes in the throat that become light, whether here it is actually a bulk closed string mode.

Case $B: h^{\prime}=0$

In this case the effective superpotential is of KKLT-type

$$
W_{\text {eff }}=\tilde{B}_{0} f+\frac{f}{2 \pi i} \hat{C} e^{-\frac{2 \pi h}{f} S}
$$

In the large $\operatorname{Re}(S)=g_{s}^{-1}$ regime that we are working in, the resulting F-term condition $F_{S}=0$ can be written as

$$
i h \hat{C} e^{-\frac{2 \pi h}{f} S}-\frac{f \tilde{B}_{0}}{2 \operatorname{Re}(\mathrm{S})}+\ldots=0
$$

Abbreviating $\tilde{s}:=\frac{2 \pi h}{f} \operatorname{Re}(S)$ and taking the values of the coefficients for the quintic (3.5) one gets for the axion $C_{0}=0$ and a transcendental relation for the saxion

$$
e^{\tilde{s}}=\frac{i \hat{C}}{\pi \tilde{B}_{0}} \tilde{s}, \quad \text { with } \quad \hat{C}=\exp (-1+2 \pi i D)
$$

where the prefactor does not depend on the fluxes but only on the coefficients appearing in the periods of the underlying CY threefold. For the quintic one obtains for the prefactor $\lambda=\frac{i \hat{C}}{\pi \tilde{B}_{0}} \sim-0.55$, a value that is way too low to admit solutions to this equation in the regime $\tilde{s}>1$. Let us now assume that we have a threefold where $\lambda \gg 1$. In that case, one would find a KKLT-like minimum with $\tilde{s}>1$ as the solution of the transcendental equation $\log \tilde{s}=\tilde{s}-\log \lambda$.

In the framework of large field inflation via axions it has been claimed that in the controllable regime the axion decay constant cannot be larger than one (in natural units where $M_{\mathrm{pl}}=1$ ). Since here we generate the exponential not directly via instantons but via moduli stabilization close to the conifold, it is interesting to investigate the appearing value of the axion decay constant. For that purpose, let us consider the scalar potential for the axion $\theta=C_{0}$ after plugging in the minimum value of $\tilde{s}$

$$
V(\theta)=\frac{1}{A \mathcal{V}^{2}(S+\bar{S})} 2\left|f \tilde{B}_{0}\right|^{2}\left[1-\cos \left(\frac{2 \pi h}{f} \theta\right)\right] .
$$

To compute the axion decay constant one has to go to the canonically normalized field $\tilde{\theta}=\theta /(\sqrt{2} \operatorname{Re}(S))$. This yields

$$
V(\tilde{\theta})=\frac{1}{A \mathcal{V}^{2} \operatorname{Re}(S)}\left|f \tilde{B}_{0}\right|^{2}[1-\cos (\sqrt{2} \tilde{s} \tilde{\theta})]
$$

so that $f=1 /(\sqrt{2} \tilde{s})<1$ in the regime where we have control. 
From (3.32) one can directly read off the mass of the axion

$$
M_{\tilde{\theta}}^{2} \sim \frac{M_{\mathrm{pl}}^{2} h f}{\mathcal{V}^{2}} \frac{4 \pi\left|\tilde{B}_{0}\right|^{2} \tilde{s}}{A}
$$

For this KKLT-like minimum of the axio-dilaton the mass of the complex structure modulus (3.20) can be simplified to

$$
M_{Z}^{2} \sim \frac{M_{\mathrm{pl}}^{2} h f}{\mathcal{V}^{2}} \frac{A}{8 \pi\left|\tilde{B}_{0}\right|^{2} \tilde{s}} .
$$

Recall that the value of $\tilde{s}$ only depends on the parameter $\lambda$. Therefore, up to CY threefold dependent data, the two masses scale in the same way with the fluxes and the overall volume. Therefore, in this case there is no exponential hierarchy among them.

\subsection{Conic LVS scenario}

So far, the scalar potential did not depend on the Kähler moduli, i.e. in particular on the overall volume of the CY. As we have seen, for the mass of the conic modulus $Z$ to remain below the string scale, we need $\mathcal{V}|Z|^{2} \gg 1$. Therefore, one needs to dynamically freeze the overall volume modulus at an exponentially large size. This makes it natural to combine our approach with the large volume scenario, reviewed in section 2.3.

Kähler moduli stabilization. Note that for the LVS minimum to exist one needs $h^{2,1}>$ $h^{1,1}$, which is clearly not satisfied for the mirror of the quintic. The minimal setup would therefore be a CY with Hodge numbers $\left(h^{2,1}, h^{1,1}\right)=(3,2)$. Close to a conifold singularity with $|Z| \ll 1$ we expect that the two additional complex structure moduli can be stabilized via fluxes at the mass scale of the axio-dilaton (3.26).

Integrating out all these moduli, we finally have to treat the issue of Kähler moduli stabilization. Since we do not explicitly know the one-loop Pfaffian, we allow it to depend polynomially on $Z$ and make the ansatz

$$
W_{\text {inst }}\left(T_{s}\right)=W_{0}+A_{s} Z^{N} e^{-a_{s} T_{s}}
$$

with $W_{0} \sim f$ and the c-number $Z \sim \hat{C} \exp \left(-\frac{2 \pi h}{f} S\right)$. Now we proceed as in section 2.3, where only the value of $A_{s}$ has been changed according to $A_{s} \rightarrow A_{s} Z^{N}$ and is now an exponentially small number. In the non-supersymmetric AdS-type large-volume minimum the Kähler moduli get now stabilized at

$$
\tau_{s}=\frac{(4 \xi)^{\frac{2}{3}}}{g_{s}}, \quad \mathcal{V}=\frac{W_{0} \xi^{\frac{1}{3}}}{2^{\frac{1}{3}} g_{s}^{\frac{1}{2}}\left|a_{s} A_{s} Z^{N}\right|} e^{a_{s} \tau_{s}}
$$

For $N>0$ the exponentially small value of $Z$ only further enhances the size of $\mathcal{V}$. For the crucial combination we thus obtain

$$
\mathcal{V}|Z|^{2} \sim \exp \left[\frac{a_{s}}{g_{s}}\left(\frac{h(N-2)}{f}+(4 \xi)^{\frac{2}{3}}\right)\right] .
$$

For $N \geq 2$ this is naturally larger than one and for $N \leq 1$ we can choose $f \gg h$ so that the exponent becomes positive. Recall from section 3.2 that there was no direct correlation between the fluxes $f$ and $h$. For staying in the perturbative regime only $h>h^{\prime}$ and $f>h^{\prime}$ was required. 


\begin{tabular}{|c|c|}
\hline Scale & $(\text { Mass })^{2}$ in $M_{\mathrm{Pl}}^{2}$ \\
\hline string scale $M_{\mathrm{s}}^{2}$ & $\frac{g_{s}^{1 / 2}}{\mathcal{V}}$ \\
Kaluza-Klein scale $M_{\mathrm{KK}}^{2}$ & $\frac{1}{\mathcal{V}^{4 / 3}}$ \\
conic modulus $M_{Z}^{2}$ & $\frac{f^{4} g_{s}^{3}}{\mathcal{V}^{2}|Z|^{2}}$ \\
small Kähler modulus $M_{\tau_{s}}^{2}$ & $\frac{f^{2}}{g_{s} \mathcal{V}^{2}}$ \\
gravitino $M_{3 / 2}^{2}$ & $\frac{f^{2} g_{s}}{\mathcal{V}^{2}}$ \\
axio-dilaton $M_{S}^{2}$ and c.s. moduli & $\frac{f^{2} g_{s}}{\mathcal{V}^{2}}$ \\
large Kähler modulus $M_{\tau_{b}}^{2}$ & $\frac{f^{2}}{g_{s}^{1 / 2} \mathcal{V}^{3}}$ \\
\hline
\end{tabular}

Table 2. Mass scales and moduli masses for the conic LVS.

Mass hierarchy. Let us now consider the moduli masses at the minimum. We observe that the masses of the Kähler moduli in (2.18) do not depend on the parameter $A_{s}$ and therefore not on $Z$. Thus, one still finds

$$
\begin{aligned}
& M_{\tau_{b}}^{2} \sim \frac{W_{0}^{2} \xi}{A g_{s}^{\frac{1}{2}} \mathcal{V}^{3}} M_{\mathrm{pl}}^{2}, \quad M_{\rho_{b}}^{2} \sim 0, \\
& M_{\tau_{s}}^{2} \sim M_{\rho_{s}}^{2} \sim \frac{a_{s}^{2} W_{0}^{2} \xi^{\frac{4}{3}}}{A g_{s} \mathcal{V}^{2}} M_{\mathrm{pl}}^{2} .
\end{aligned}
$$

The gravitino mass is given by

$$
M_{3 / 2}^{2}=e^{K}|W|^{2} \sim \frac{g_{s} W_{0}^{2}}{A \mathcal{V}^{2}} M_{\mathrm{Pl}}^{2} .
$$

In table 2 we summarize all relevant mass scales, where we only displayed the dependence on $\mathcal{V}, g_{s}, Z$ and the large flux $f$. We choose $h^{\prime} \neq 0$ to determine the axio-dilaton mass $M_{S}$ and ordered the mass scales in the perturbative large $\mathcal{V}$ and small $g_{s},|Z|$ regime.

Hence, we obtain the mass hierarchy

$$
M_{\tau_{b}}<M_{\tau_{s}} \sim M_{S}<M_{Z}<M_{\mathrm{KK}}<M_{\mathrm{S}}<M_{\mathrm{Pl}}
$$

Since the mass of the modulus $\tau_{s}$ is of the same volume order $1 / \mathcal{V}^{2}$ as the axio-dilaton $S$, one might worry whether integrating out $S$ and only considering the Kähler moduli is actually justified. However, as shown in [3] the minimum above remains a minimum of the full potential including axio-dilaton and complex structure moduli. The reason is that these moduli are flux-stabilized and enter the scalar potential already at the order $\mathcal{O}\left(\frac{1}{\mathcal{V}^{2}}\right)$ 
that, in the large volume regime, dominate over the terms of order $\mathcal{O}\left(\frac{1}{\mathcal{V}^{3}}\right)$ by which the Kähler moduli are stabilized.

We conclude that flux stabilization of the complex structure and the axio-dilaton close to the conifold singularity can be consistently combined with the LVS scenario, thus guaranteeing a reliable effective field theory approach where warping is negligible. The masses of the moduli get split up so that one gains parametric control over their ratios.

\section{Towards axion alignment}

In this section we generalize the previous set-up to models with more complex structure moduli. The motivation is twofold. First, we would like to see whether the periods of threefolds with more complex structure moduli still arrange themselves to provide Kähler potentials with shift-symmetries. Second, with more complex structure moduli we will be able to address recent questions about large field inflation. Concretely, we will design a model of aligned inflation according to the proposal of [41]. There one linear combination of two axions was stabilized via fluxes by polynomial terms in $W$, while the orthogonal direction was stabilized by exponentially subleading terms in $W$. This provides another possible application of the exponential terms in the effective superpotential after integrating out the most heavy complex structure modulus $Z$. Models with the inflaton being identified with an axion-like complex structure modulus have also been investigated in [25, 26, 33, 34].

\subsection{Periods for $\mathbb{P}_{11226}[12]$}

Similar to the quintic, we now numerically evaluate the periods of the Calabi-Yau threefold $\mathbb{P}_{11226}[12]^{(128,2)}$ in the vicinity of the conifold locus. We are particularly interested in computing the Kähler potential to see whether it exhibits any shift-symmetry.

The mirror of the above manifold has two complex structure moduli that appear as deformations of the hypersurface constraint

$$
P=z_{1}^{12}+z_{2}^{12}+z_{3}^{6}+z_{4}^{6}+z_{5}^{2}-12 \psi z_{1} z_{2} z_{3} z_{4} z_{5}-2 \phi z_{1}^{6} z_{2}^{6} .
$$

The codimension one conifold locus is at $864 \psi^{6}+\phi=1$. In the regime of small $\psi$ and $\phi$ the fundamental period is given by $[42,43]$

$$
\varpi_{f}(\psi, \phi)=-\frac{1}{6} \sum_{n=1}^{\infty} \frac{\Gamma\left(\frac{n}{6}\right)(-12 \psi)^{n} u_{-\frac{n}{6}}(\phi)}{\Gamma(n) \Gamma^{2}\left(1-\frac{n}{6}\right) \Gamma\left(1-\frac{n}{2}\right)}
$$

with

$$
u_{-\frac{n}{6}}(\phi)=\frac{e^{-i \pi \frac{n}{12}}}{2 \Gamma\left(\frac{n}{6}\right)} \sum_{m=0}^{\infty} \frac{e^{i \pi \frac{m}{2}} \Gamma\left(\frac{m}{2}+\frac{n}{12}\right)(2 \phi)^{m}}{m ! \Gamma\left(1-\frac{m}{2}-\frac{n}{12}\right)}
$$

These expressions are valid for $|\phi|<1$ and $\left|\frac{864 \psi^{6}}{\phi \pm 1}\right|<1$. A basis of periods solving the Picard-Fuchs equations can be derived from $\varpi_{f}$ via

$$
\varpi_{i}(\psi, \phi)=-\frac{(2 \pi i)^{3}}{\psi} \varpi_{f}\left(\lambda^{i} \psi, \lambda^{6 i} \phi\right)
$$


with $i=0, \ldots, 5$ and $\lambda=\exp (\pi i / 6)$. These periods do not yet form a symplectic basis. The latter was determined in $[44,45]$ as

$$
\left(\begin{array}{l}
F_{0} \\
F_{1} \\
F_{2} \\
X^{0} \\
X^{1} \\
X^{2}
\end{array}\right)=\left(\begin{array}{cccccc}
\frac{3}{2} & \frac{3}{2} & \frac{1}{2} & \frac{1}{2} & -\frac{1}{2} & -\frac{1}{2} \\
-1 & 1 & 0 & 0 & 0 & 0 \\
1 & 0 & 1 & 0 & 0 & 0 \\
-\frac{1}{2} & 0 & \frac{1}{2} & 0 & \frac{1}{2} & 0 \\
1 & 0 & 0 & 0 & 0 & 0 \\
\frac{1}{2} & \frac{1}{2} & -\frac{1}{2} & \frac{1}{2} & -\frac{1}{2} & \frac{1}{2}
\end{array}\right)\left(\begin{array}{l}
\varpi_{0} \\
\varpi_{1} \\
\varpi_{2} \\
\varpi_{3} \\
\varpi_{4} \\
\varpi_{5}
\end{array}\right)
$$

We now want to evaluate these periods close to the conifold locus, which lies on the boundary of validity of the expansion (4.2). Concretely, we restrict to the region around the point $\psi=\psi_{0}=864^{-\frac{1}{6}}$ and $\phi=0$ on the conifold locus. Writing $\psi=\psi_{0}+\xi$, we numerically evaluate the periods up to quadratic order in $(\xi, \phi)$. This has also been done up to linear order in [6], but not to the level of accuracy that we need for our purposes. The numerical computation of the sum in (4.2) up to $n \sim 20000$ gives $^{3}$

$$
\begin{aligned}
F_{0}= & 4323.04 i-1548.4 i \xi+107.7 i \phi-3893.22 i \xi^{2}-278.46 i \xi \phi+27.78 i \phi^{2}, \\
F_{1}= & 3191 \xi+172.29 \phi+7583.59 \xi^{2}-533.36 \xi \phi+65.2 \phi^{2}, \\
F_{2}= & (-492.72+1976.76 i)+(372.45-302.3 i) \xi-(258.97+58.87 i) \phi \\
& -(436.95-262.39 i) \xi^{2}-(6.5+14.41 i) \xi \phi-(3.09-24.14 i) \phi^{2}
\end{aligned}
$$

and

$$
\begin{aligned}
X^{0}= & -(994.58+184.76 i)+(859.48+471.9 i) \xi+(10.04-112.7 i) \phi \\
& +(1831.84+2209 i) \xi^{2}-(136.09-124.82 i) \xi \phi+(3.13+10.25 i) \phi^{2}, \\
X^{1}= & -\frac{1}{2 \pi i} F_{1} \log F_{1}+784.36 i-4997.53 i \xi-185.8 i \phi+\ldots, \\
X^{2}= & 369.52 i-943.81 i \xi+225.4 i \phi-4418 i \xi^{2}-249.64 i \xi \phi-20.5 i \phi^{2} .
\end{aligned}
$$

Note that the order two terms in $X^{1}$ will not be relevant for computing the Kähler potential up to quadratic order. Next, we go to inhomogeneous coordinates where $F_{0}=1$ and substitute

$$
\phi \rightarrow-18.52 \xi+25.09 i Z-231.17 \xi^{2}+408.85 i \xi Z+222.58 Z^{2}
$$

followed by a second substitution

$$
\xi \rightarrow 1.97 Y+1.13 i Z-62.84 Y^{2}-8.7 i Z Y+4.07 Z^{2}
$$

\footnotetext{
${ }^{3}$ For some of these numbers we gained a better precision, which was then also used in the following computations.
} 
Finally, in terms of the fields $Z$ and $Y$, up to quadratic order, the periods take the form

$$
\begin{aligned}
F_{0}= & 1 \\
F_{1}= & Z \\
F_{2}= & (0.46+0.11 i)+(1.10-2.17 i) Y-0.19 Z \\
& -(7.34-14.73 i) Y^{2}+(2.71+1.42 i) Y Z+(0.11-1.69 i) Z^{2}
\end{aligned}
$$

and

$$
\begin{aligned}
X^{0}= & (-0.04+0.23 i)+(1.10+0.06 i) Y+0.17 Z \\
& -(7.34+1.83 i) Y^{2}+(0.55+1.42 i) Y Z+(0.11-0.17 i) Z^{2}, \\
X^{1}= & -\frac{1}{2 \pi i} Z \log Z+0.18-0.42 Y-1.43 i Z+\ldots, \\
X^{2}= & 0.09-2.19 Y+14.67 Y^{2}-2.84 i Y Z-0.22 Z^{2} .
\end{aligned}
$$

Here we have set a numerical number to zero, if it vanishes up to the order $O\left(10^{-4}\right)$. Moreover, the numbers appearing in the periods are not unrelated. Up to order $O\left(10^{-4}\right)$, we find e.g.

$$
\begin{aligned}
2 X^{0}+X^{2} & =0.46 i+0.13 i Y+0.33 Z-3.66 i Y^{2}+1.11 Y Z-0.34 i Z^{2} \\
F_{2}-X^{0} & =(0.5-0.11 i)-2.24 i Y-0.36 Z+16.56 i Y^{2}+2.16 Y Z-1.52 i Z^{2} .
\end{aligned}
$$

Due to these relations, the resulting Kähler potential at linear order simplifies considerably

$$
\begin{aligned}
K_{\mathrm{cs}} & =-\log \left[-i \Pi^{\dagger} \Sigma \Pi\right] \\
& =-\log \left[\frac{1}{2 \pi}|Z|^{2} \log \left(|Z|^{2}\right)+A+\operatorname{Re} Y+B(\operatorname{Re} Y)^{2}+C|Z|^{2} \cdots\right]
\end{aligned}
$$

with $A=0.44$ and $B=-19.05$ and $C=-2.86$. As for the quintic, the Kähler potential exhibits the shift symmetry $Z \rightarrow e^{i \theta} Z$ and in addition the shift symmetry $\operatorname{Im}(Y) \rightarrow$ $\operatorname{Im}(Y)+\theta$. Therefore, in this regime close to the conifold, $\operatorname{Im}(Y)$ behaves like an axion.

\subsection{Freezing axio-dilaton and complex structures}

Motivated by the previous periods and the shift-symmetric Kähler potential we analyze the resulting supergravity models for moduli stabilization. Here we proceed analogously to section 3 and first stabilize the $Z$ modulus by the usual combination of R-R and NSNS fluxes on the two cycles related to the conifold singularity. Integrating out this most heavy complex structure modulus leads to effective Kähler- and superpotentials that we subsequently study in a more phenomenological manner, i.e. without reference to a concrete CY threefold. Thus, we allow us to be more flexible with independent fluxes and numerical prefactors than an actual threefold example may permit.

For such an effective model with two complex structure moduli $(Z, Y)$, up to linear order in $Y$, we make the ansatz

$$
K_{\mathrm{eff}}=-2 \log \mathcal{V}-\log (S+\bar{S})-\log \left(A+\frac{1}{2}(Y+\bar{Y})\right)
$$


and

$$
W_{\text {eff }}=f \alpha+h^{\prime} \beta S+\hat{f}^{\prime} \gamma Y
$$

with $\alpha, \beta, \gamma \in \mathbb{C}$ and $A \in \mathbb{R}$. However, the minimum conditions $D_{S} W=D_{Y} W=0$ only admit solutions with $\operatorname{Re}(S)=0$, i.e. in the unphysical domain. Therefore, our ansatz is not yet sufficiently generic.

Adding the second order term to the Kähler potential, we make the ansatz

$$
\begin{aligned}
K_{\mathrm{eff}} & =-2 \log \mathcal{V}-\log (S+\bar{S})-\log \left(A+\kappa \operatorname{Re} Y-(\operatorname{Re} Y)^{2}\right) \\
W_{\mathrm{eff}}^{(0)} & =i \alpha\left(f+h^{\prime} S+\hat{f}^{\prime} Y\right)
\end{aligned}
$$

for the effective Kähler- and superpotential, with the model dependent order one parameters $A, \alpha, \kappa \in \mathbb{R}$. Just for simplicity, we were choosing $\alpha=\beta=\gamma$. Including just these single first order terms in $W_{\text {eff }}$ implicitly means that we have assumed that possible higher order polynomial terms like $Y^{n}$ are either not present or are subleading. Whether the concrete form of realistic periods admit such a choice remains to be seen.

For the model (4.16) we find a Minkowski minimum at

$$
\begin{gathered}
\Sigma=\hat{f}^{\prime} \zeta_{0}+h^{\prime} c_{0}=0 \\
s=s_{0}=\frac{1}{h^{\prime}} \sqrt{f^{2}-A \hat{f}^{\prime 2}+\kappa f \hat{f}^{\prime}} \\
\operatorname{Re}(Y)=y_{0}=\frac{1}{\hat{f}^{\prime}}\left(-f+\sqrt{f^{2}-A \hat{f}^{\prime 2}+\kappa f \hat{f}^{\prime}}\right) .
\end{gathered}
$$

where $Y=y+i \zeta$. Note that for $f / \hat{f}^{\prime} \gg 1$ these expressions simplify drastically

$$
\Sigma=0, \quad s_{0}=\frac{f}{h^{\prime}}, \quad y_{0}=\frac{\kappa}{2}+O\left(\frac{\hat{f}^{\prime}}{f}\right) .
$$

Therefore, for $f / h^{\prime} \gg 1$ and $\kappa<1$ the values of the moduli lie in the perturbative regime of small string coupling and small complex structure modulus $Y$. In the following, we analyze this model in more detail with special focus on the axion sector. For simplicity we choose $\kappa=0$ so that

$$
\Sigma=0, \quad s_{0}=\frac{f}{h^{\prime}}, \quad y_{0}=-\frac{A \hat{f}^{\prime}}{2 f}+O\left(\left(\hat{f}^{\prime} / f\right)^{2}\right) .
$$

Note that the second axion $\Theta$ is still massless at this level. It is clear that once additional terms like e.g. $\Delta W=i B Y^{2}$ are present in the superpotential, $\Theta$ also gets stabilized with a mass that is governed by the parameter $B$. For $B$ of order one, all four fields will have the same order of masses, but for a parametrically smaller value of $B$ the axion $\Theta$ will be the lightest state of all complex structure and axio-dilaton moduli.

Recall that, after integrating out $Z$, one also gets an exponential term like $\exp \left(-\frac{2 \pi}{f} h S\right)$ in $W$. As its size depends on a different modulus, this term can in principle compete 
with the higher order polynomial terms $Y^{n}$. For illustrative purposes, let us consider in the following section the possible moduli stabilization scheme, once we include only this exponential term in $W$ and assume that polynomial terms are either absent or subleading. We understand that this is a very strong assumption that needs to be tested for concrete CY manifolds (e.g. along the line reported in [34]).

\subsection{Axion alignment}

As we have seen, only one linear combination of the two axions is stabilized by terms appearing linearly in $W$. We now analyse the phenomenological effective supergravity model (4.16)

$$
\begin{aligned}
W_{\mathrm{eff}} & =W_{\mathrm{eff}}^{(0)}+W_{\mathrm{eff}}^{(1)} \\
& =i \alpha\left(f+h^{\prime} S+\hat{f}^{\prime} Y\right)+\frac{f \hat{C}}{2 \pi i} \exp \left(-\frac{2 \pi}{f}(h S+\hat{f} Y)\right) .
\end{aligned}
$$

Here we have included a term $\exp \left(-\frac{2 \pi}{f} \hat{f} Y\right)$ which is induced from a coupling $\hat{f} Z Y$ in the original superpotential.

Let us now derive an effective scalar potential $V_{\text {eff }}$ for the so far unstabilized axion appearing in the exponent of (4.20). If we naively integrate out the already stabilized moduli via

$$
\begin{aligned}
& \left.D_{S} W_{\text {eff }}\right|_{S_{0}, Y_{0}}=c_{S}|Z| \exp \left(-\frac{2 \pi i}{f} \Theta\right), \\
& \left.D_{Y} W_{\text {eff }}\right|_{S_{0}, Y_{0}}=c_{Y}|Z| \exp \left(-\frac{2 \pi i}{f} \Theta\right)
\end{aligned}
$$

with $\Theta=(h c+\hat{f} \zeta)$ and $c_{S}, c_{Y} \neq 0$, we realize that $\left.V_{\text {eff }}\right|_{S_{0}, Y_{0}}$ does not depend on the axion $\Theta$ and is non-vanishing at order $O\left(|Z|^{2}\right)$. However, this is not what one expects. In the true vacuum (at order $O\left(|Z|^{2}\right)$ ) the vacuum energy should be vanishing and the remaining axion $\Theta$ should be stabilized at $\Theta=0$.

Indeed to see this, we first have to take the backreaction of the exponential term on the stabilization of the saxions into account. Perturbing around the leading order values $y_{0}, s_{0}$ by $\Delta y_{0} \sim O(|Z|)$ and $\Delta s_{0} \sim O(|Z|)$, we fix $\Delta y_{0}$ and $\Delta s_{0}$ by requiring $\left.D_{S} W_{\text {eff }}\right|_{S_{0}, Y_{0}, \Theta=0}=$ $\left.D_{Y} W_{\text {eff }}\right|_{S_{0}, Y_{0}, \Theta=0}=0+O\left(|Z|^{2}\right)$. For $\kappa=0, f / \hat{f}^{\prime} \gg 1$ and at leading non-vanishing order in $|Z|$ we find

$$
\Delta s_{0} \sim-\frac{f}{2 \pi \alpha h^{\prime}}\left(1+\frac{4 \pi h}{h^{\prime}}\right)|Z|, \quad \Delta y_{0} \sim-\frac{A \hat{f}}{2 \alpha f}|Z|
$$

This backreaction induced shift of the vacuum leads to the correct effective scalar potential

$$
\begin{aligned}
& V_{1}=G^{S \bar{S}} D_{S} W D_{\bar{S}} \bar{W}=\frac{|Z|^{2}}{2 \pi^{2}} f^{2}\left(1+\frac{4 \pi h}{h^{\prime}}\right)^{2}\left(1-\cos \left(\frac{2 \pi}{f} \Theta\right)\right) \\
& V_{2}=G^{Y \bar{Y}} D_{Y} W D_{\bar{Y}} \bar{W}=\frac{|Z|^{2} A}{4 \pi^{2}} \hat{f}^{\prime 2}\left(1+\frac{4 \pi \hat{f}}{\hat{f}^{\prime}}\right)^{2}\left(1-\cos \left(\frac{2 \pi}{f} \Theta\right)\right),
\end{aligned}
$$



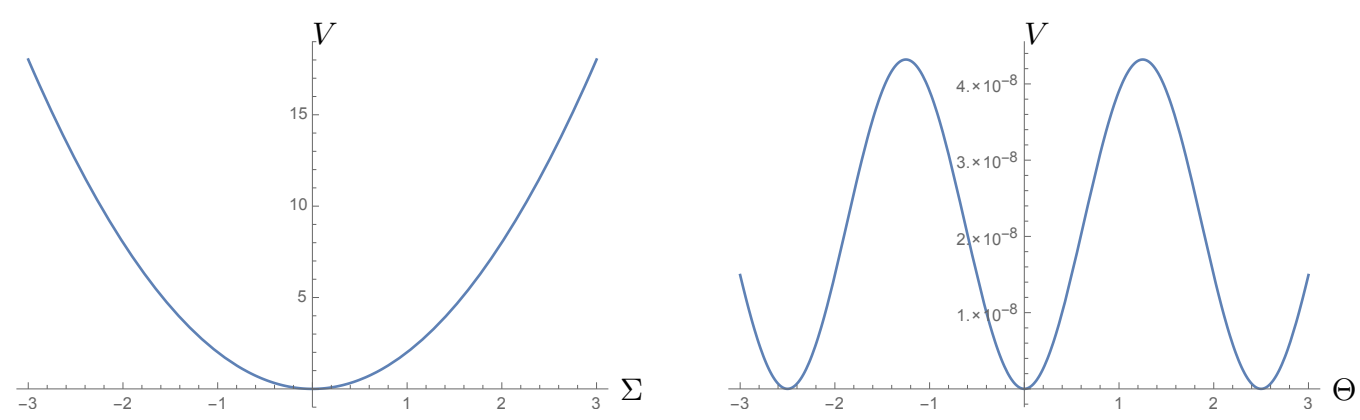

Figure 1. Scalar potential for the two axions $\Sigma$ and $\Theta$ for $f=10, h^{\prime}=\hat{f}^{\prime}=1, h=-\hat{f}=2$, $\hat{C}=1$ and $A=0.1$.

so that in the regime $f / \hat{f}^{\prime} \gg 1$ and $h / h^{\prime} \gg 1$ we eventually obtain the effective potential for $\Theta$

$$
V_{\text {eff }}=e^{K_{\text {eff }}}\left(V_{1}+V_{2}\right) \sim \frac{4|Z|^{2}}{A \mathcal{V}^{2}} \frac{f h^{2}}{h^{\prime}}\left(1-\cos \left(\frac{2 \pi}{f} \Theta\right)\right)
$$

Note that the potential exhibits the expected Minkowski minimum at $\Theta=0$.

As a check of our approach, in figure 1 we have plotted the axion dependence of the full scalar potential for the saxions fixed at the Minkowski minimum. It nicely shows the periodic form of the potential (4.24) and that the height of the potential in $\Theta$ direction is hierarchically smaller than in $\Sigma$ direction.

In order to apply the model at hand to axion inflation, it remains to rewrite the potential in terms of the canonically normalized field $\tilde{\Theta}$ which we then employ as inflaton. Due to the diagonal structure of the Kähler metric, one finds in the regime $f / f^{\prime} \gg 1$

$$
\tilde{\Theta}=\frac{h^{\prime}}{\sqrt{A} \hat{f}^{\prime}} \Theta
$$

such that the canonically normalized inflaton potential is given by

$$
V_{\mathrm{eff}}=\frac{4|Z|^{2}}{A \mathcal{V}^{2}} \frac{f h^{2}}{h^{\prime}}\left(1-\cos \left(\frac{2 \pi \sqrt{A}\left(h \hat{f}^{\prime}-h^{\prime} \hat{f}\right)}{f h^{\prime}} \tilde{\Theta}\right)\right) \equiv V_{0}\left(1-\cos \left(\frac{\tilde{\Theta}}{f_{\tilde{\Theta}}}\right)\right) .
$$

The axion decay constant $f_{\tilde{\Theta}}$ signalizes the appearance of an alignment mechanism as

$$
f_{\tilde{\Theta}}=\frac{f}{2 \pi \sqrt{A}} \frac{h^{\prime}}{h \hat{f}^{\prime}-h^{\prime} \hat{f}} .
$$

By aligning the fluxes as $\left(h \hat{f}^{\prime}-h^{\prime} \hat{f}\right)<h^{\prime}$ we can obtain an axion decay constant larger than one. This is very reminiscent of the KNP-axion alignment mechanism [19], the main difference being that one linear combination of axions is fixed by fluxes at linear order in the fields and only the second combination by instanton-like terms.

Coming back to our assumptions about the suppression of polynomial terms in $W$, we consider the superpotential

$$
W_{\mathrm{eff}}=i \alpha\left(f+h^{\prime} S+\hat{f}^{\prime} Y\right)+i B Y^{2}+\frac{f \hat{C}}{2 \pi i} \exp \left(-\frac{2 \pi}{f}(h S+\hat{f} Y)\right)
$$



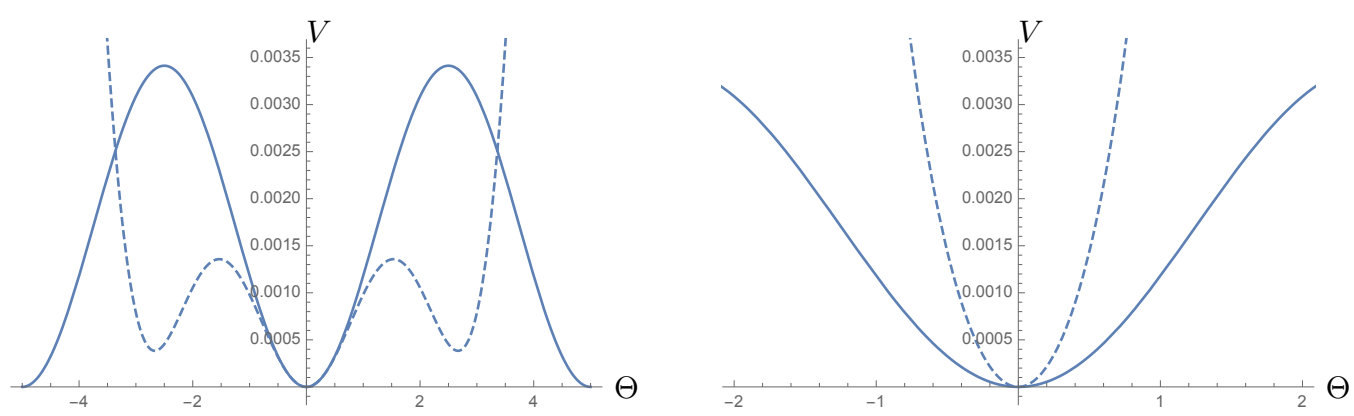

Figure 2. Scalar potential (dashed lines) for the axion $\Theta$ for $f=10, h^{\prime}=\hat{f}^{\prime}=1, h=-\hat{f}=1$, $\hat{C}=1, A=0.1$ and $B=0.01$ in the left-handed plot and $B=0.1$ in the right-handed plot. For comparison, the solid lines show the potential for $B=0$.

and in figure 2 display the new effective potential for $\Theta$ (by dashed lines) for two different values of the parameter $B$.

The left-handed plot shows that here $B$ is sufficiently small so that in the direct neighborhood of $\Theta=0$ the potential is dominated by the exponential term. Whereas, in the right-handed plot $B$ is so large that the mass of $\Theta$ comes from the quadratic term. As said, we are not claiming that higher order terms are really suppressed for a concrete CY, but just want to show which kind of scenarios are in principle possible for moduli stabilization close to the conifold. We would not be surprised if once again a concrete string theoretic proposal for realizing large field inflation fails as one loses control over certain dangerous terms. In this spirit we proceed with discussing the purely exponential case.

A comment on the WGC. Even though the effective exponential terms in the superpotential do not directly arise from instanton contributions, one can ask whether they satisfy a generalized version of the weak gravity conjecture. For instantons the conjecture says that the product of the instanton action times the axion decay constant has to be smaller than one

$$
S_{\text {inst }} f_{\text {inst }} \leq 1
$$

If this is to be satisfied for the instanton with the lowest action, one has the strong version of the WGC. Apparently, this is violated for the aligned axion $\tilde{\Theta}$ whose action can be written as

$$
S_{\text {inst }}=\frac{2 \pi}{f}\left(h s_{0}+\hat{f} y_{0}\right) \sim \frac{2 \pi}{f} h s_{0} \sim \frac{2 \pi h}{h^{\prime}},
$$

so that in the case of alignment one gets

$$
S_{\text {inst }} f_{\tilde{\Theta}} \sim \frac{f h}{\sqrt{A}\left(h \hat{f}^{\prime}-h^{\prime} \hat{f}\right)}>1 .
$$

The mild form of the WGC says that for an axion with decay constant $f_{\tilde{\Theta}}$ there is some instanton with instanton action $S_{\text {inst }}^{(2)}$ satisfying the WGC condition. Therefore, to satisfy 
the mild WGC, it is sufficient to have a situation where the contribution of these two instantons reads as

$$
V \sim e^{-2 S_{\text {inst }}}\left(1-\cos \left(\frac{\tilde{\Theta}}{f_{\tilde{\Theta}}}\right)\right)+e^{-2 S_{\text {inst }}^{(2)}}\left(1-\cos \left(\frac{k \tilde{\Theta}}{f_{\tilde{\Theta}}}\right)\right),
$$

with $k \in \mathbb{Z}$. For sufficiently large $k$, the axion decay constant $f_{\tilde{\Theta}} / k$ can be sub-Planckian, while for $S_{\text {inst }}<S_{\text {inst }}^{(2)}$ the first term is still dominant and enables inflation. This loop-hole was pointed out in $[29,31]$ and has been realized for complex structure aligned inflation in [41].

In our case the situation is similar, where the second exponential contribution could arise from a single $D(-1)$-instanton. Its action and decay constant is

$$
S_{D(-1)}=2 \pi s_{0}=\frac{2 \pi f}{h^{\prime}}, \quad f_{D(-1)}=\frac{h^{\prime}}{2 \pi \sqrt{A} \hat{f}^{\prime}}=\frac{f_{\tilde{\Theta}}}{k}
$$

with $k=\left(f \hat{f}^{\prime}\right) /\left(h \hat{f}^{\prime}-h^{\prime} \hat{f}\right)$. With the denominator being equal to one in the case of alignment, $k$ is a large integer. Moreover, for $f / h>1$ the $D(-1)$ instanton action is subleading and inflation can still occur.

Mass scales. Let us finally compute the different mass scales to confirm our various effective approaches and in particular justify integrating out massive fields at several stages of our computation. Let us denote by $M_{\text {mod }}$ the masses of the moduli $\Sigma, s$ and $\operatorname{Re}(Y)$ in the minimum (4.19). Up to numerical prefactors of order $O(1)$, the mass eigenvalues of the canonically normalized mass matrix $\left(M^{2}\right)^{i}{ }_{j}=\frac{1}{2} G^{i k} \partial_{k} \partial_{j} V$ are given by

$$
M_{\text {mod }}^{2}=\frac{f^{2} g_{s}}{A \mathcal{V}^{2}} \text {. }
$$

In addition one can then read off the mass of the canonically normalized inflaton $\tilde{\Theta}$ from $V_{\text {eff }}$ in equation (4.26)

$$
M_{\tilde{\Theta}}^{2}=\frac{V_{0}}{f_{\tilde{\Theta}}^{2}} M_{\mathrm{Pl}}^{2} \sim \frac{|Z|^{2}}{f \mathcal{V}^{2}} M_{\mathrm{Pl}}^{2} .
$$

Hence the mass of the inflaton is exponentially suppressed relative to the mass of the other moduli stabilized at tree-level. Recall that the latter are also exponentially suppressed relative to the conic complex structure modulus $Z$, such that we obtain a mass hierarchy of the form

$$
M_{\tilde{\Theta}}<M_{\bmod }<M_{Z} .
$$

The mass scale of inflation can be read off from (4.26) as

$$
M_{\mathrm{inf}}^{2} \sim V_{0}^{\frac{1}{2}} \sim \frac{f^{\frac{1}{2}}|Z|}{\mathcal{V}}
$$

Therefore, with $g_{s} \sim 1 / f$ we obtain

$$
\frac{M_{\text {inf }}^{2}}{M_{\text {mod }}^{2}} \sim \frac{\left(\mathcal{V}|Z|^{2}\right)}{f^{\frac{1}{2}}|Z|} \quad \text { and } \quad \frac{M_{\text {inf }}^{2}}{M_{Z}^{2}} \sim\left(\mathcal{V}|Z|^{2}\right) \frac{|Z|}{f^{\frac{1}{2}}}
$$




\begin{tabular}{|c|c|}
\hline Scale & $(\mathrm{Mass})^{2}$ in $M_{\mathrm{Pl}}^{2}$ \\
\hline string scale $M_{\mathrm{s}}^{2}$ & $\frac{1}{f^{1 / 2} \mathcal{V}}$ \\
\hline Kaluza-Klein scale $M_{\mathrm{KK}}^{2}$ & $\frac{1}{\mathcal{V}^{4 / 3}}$ \\
\hline conic c.s. modulus $M_{Z}^{2}$ & $\frac{f}{\mathcal{V}^{2}|Z|^{2}}$ \\
\hline inflationary mass scale $M_{\mathrm{inf}}^{2}$ & $\frac{f^{1 / 2}|Z|}{\mathcal{V}}$ \\
\hline other moduli $M_{\bmod }^{2}$ & $\frac{f}{\mathcal{V}^{2}}$ \\
\hline gravitino mass $M_{3 / 2}^{2}$ & $\frac{f}{\mathcal{V}^{2}}$ \\
\hline large Kähler modulus $M_{\tau_{b}}^{2}$ & $\frac{f^{5 / 2}}{\mathcal{V}^{3}}$ \\
\hline inflaton $M_{\tilde{\Theta}}^{2}$ & $\frac{|Z|^{2}}{f \mathcal{V}^{2}}$ \\
\hline
\end{tabular}

Table 3. Moduli masses and scales with $g_{s} \sim 1 / f$.

so that in the regime $\mathcal{V}|Z|^{2} \gg 1$ the inflationary scale is larger than the moduli masses, but for sufficiently small $|Z|$ can be lower than the mass of the conic complex structure modulus. Therefore, for correctly describing the dynamics in the slow-rolling phase one can use the effective four-dimensional SUGRA theory after integrating out the conic modulus $Z$. The backreaction of the inflaton on the remaining moduli is expected to lead to a (welcomed) flattening of the quadratic inflaton potential [46]. Note that in this respect this model behaves better than the ones constructed in the framework of the flux scaling scenario [27], where generically the inflationary mass scale was even larger than the KK scale.

So far we did not stabilize the Kähler moduli for this inflationary model. Let us now assume that we can employ the large volume scenario and estimate the appearing mass scales as in section 3.3. We ignore possible subtleties about the order of integrating out for the moment. Since the non-supersymmetric LVS minimum is of AdS type, we also have to assume a proper uplift mechanism. As a first rough estimate, in table 3 we list all relevant mass scales.

From the table we extract the relation

$$
\frac{M_{\tilde{\Theta}}^{2}}{M_{\tau_{b}}^{2}} \sim \frac{\mathcal{V}|Z|^{2}}{f^{7 / 2}}
$$

so that in the reliable SUGRA regime with $\mathcal{V}|Z|^{2} \gg 1$ we generically find that the large Kähler modulus is lighter than the potential inflaton. Of course this spoils single field inflation and reflects a problem that seems to be very generic for complex structure moduli 
inflation $[25,26]$. One can derive the relation

$$
\frac{M_{\tilde{\Theta}}^{2}}{M_{\tau_{b}}^{2}} \sim \frac{M_{\mathrm{S}}^{2}}{f^{2} M_{Z}^{2}}
$$

so that in principle for $M_{\mathrm{s}} / M_{Z} \sim 5-8$ one can get that the axion $\tilde{\Theta}$ is the lightest mode for $f \sim 10$. Of course here one is at the boundary of control and numerical factors matter. Thus, the generic hierarchy of scales is of the form

$$
M_{\tau_{b}}<M_{\tilde{\Theta}}<M_{\text {mod }}<M_{\text {inf }} \sim M_{Z}<M_{\mathrm{KK}}<M_{\mathrm{S}}<M_{\mathrm{Pl}},
$$

guaranteeing parametric control over the mass scales in our effective SUGRA description. It is not excluded that by a certain choice of the flux $f$ one can get that the axion is in principle the lightest mode. In this case a more detailed analysis is necessary, as one cannot first stabilize the axion $\tilde{\Theta}$ and then integrate out the Kähler moduli.

Summarizing, in this corner of the string theory landscape we managed to design a string motivated effective supergravity model that features an alignment mechanism providing an axion that has an effective decay constant larger than one. The axion sector still satisfies the mild form of the WGC. However, this model will probably fail at the end, as we had to make very strong assumptions about higher order polynomial corrections to $W$ and because it is not yet clear whether one can get the Kähler moduli heavier than the inflaton.

\section{Conclusions}

In this paper we studied moduli stabilization close to the conifold locus in the complex structure moduli space. This is an attractive corner of the string theory landscape, as the Kähler potential features axionic shift symmetries that are interesting for string theory realizations of large field inflation.

Following the early ideas of GKP, after computing the periods close the conifold locus, by turning on appropriate fluxes, we managed to dynamically stabilize the conic complex structure modulus at $|Z| \ll 1$ and the other complex structure moduli and the axio-dilaton in their perturbative regime. As a self-consistency condition for the use of the GVW effective field theory we found that the total volume must be exponentially large precisely guaranteeing the absence of significant warping. We showed that a combination with the large volume scenario is possible, providing a consistent hierarchy of mass scales.

Moreover, we found that even in the absence of warping certain modes still show exponential mass hierarchies, that can be traced back to the appearance of log-terms in the periods. Let us emphasize that these are not the modes whose mass gets red-shifted in the region of strong warping. After integrating out the heavy conic complex structure modulus, one generates exponential, instanton-like terms in the superpotential. As demonstrated, these can be further exploited for moduli stabilization.

Being equipped with new mechanisms to generate exponential hierarchies we approached the important problem of designing a string theory motivated model of axion 
inflation featuring consistent moduli stabilization. Ignoring first the Kähler moduli and following the main idea of [41] we managed to construct a model including an aligned axion with axion decay constant larger than one. However, in the construction quite strong assumptions about the size of higher order corrections were made and we were not able to also freeze the Kähler moduli via the LVS such that we really end up with a fully controllable model of single field inflation. It would be interesting to see whether, as reported in [34], the higher order corrections to the periods do spoil some of our findings.

It is not clear to us whether those effective exponential terms are related to true instanton contributions on the mirror side and whether a form of the weak-gravity conjecture applies to them. Recall that they only became visible after integrating out the conic modulus $Z$. Thus, they might indeed provide a loop-hole in the axionic version of the WGC.

Finally, let us just state that the landscape is rich and still might contain new mechanisms for hierarchical moduli stabilization that are waiting to be exploited for various applications in string phenomenology and string cosmology.

\section{Acknowledgments}

We are grateful to Shanta de Alwis, Anamaria Font, Thomas Grimm, Kepa Sousa and Irene Valenzuela for discussions and to Michael Fuchs for collaboration during early stages of this work.

Open Access. This article is distributed under the terms of the Creative Commons Attribution License (CC-BY 4.0), which permits any use, distribution and reproduction in any medium, provided the original author(s) and source are credited.

\section{References}

[1] S.B. Giddings, S. Kachru and J. Polchinski, Hierarchies from fluxes in string compactifications, Phys. Rev. D 66 (2002) 106006 [hep-th/0105097] [INSPIRE].

[2] S. Kachru, R. Kallosh, A.D. Linde and S.P. Trivedi, De Sitter vacua in string theory, Phys. Rev. D 68 (2003) 046005 [hep-th/0301240] [INSPIRE].

[3] V. Balasubramanian, P. Berglund, J.P. Conlon and F. Quevedo, Systematics of moduli stabilisation in Calabi-Yau flux compactifications, JHEP 03 (2005) 007 [hep-th/0502058] [INSPIRE].

[4] I.R. Klebanov and M.J. Strassler, Supergravity and a confining gauge theory: duality cascades and chi SB resolution of naked singularities, JHEP 08 (2000) 052 [hep-th/0007191] [INSPIRE].

[5] S. Ashok and M.R. Douglas, Counting flux vacua, JHEP 01 (2004) 060 [hep-th/0307049] [INSPIRE].

[6] J.P. Conlon and F. Quevedo, On the explicit construction and statistics of Calabi-Yau flux vacua, JHEP 10 (2004) 039 [hep-th/0409215] [INSPIRE].

[7] S. Gukov, C. Vafa and E. Witten, CFT's from Calabi-Yau four folds, Nucl. Phys. B 584 (2000) 69 [Erratum ibid. B 608 (2001) 477] [hep-th/9906070] [INSPIRE]. 
[8] T.R. Taylor and C. Vafa, RR flux on Calabi-Yau and partial supersymmetry breaking, Phys. Lett. B 474 (2000) 130 [hep-th/9912152] [INSPIRE].

[9] O. DeWolfe and S.B. Giddings, Scales and hierarchies in warped compactifications and brane worlds, Phys. Rev. D 67 (2003) 066008 [hep-th/0208123] [INSPIRE].

[10] S.P. de Alwis, On potentials from fluxes, Phys. Rev. D 68 (2003) 126001 [hep-th/0307084] [INSPIRE].

[11] S.B. Giddings and A. Maharana, Dynamics of warped compactifications and the shape of the warped landscape, Phys. Rev. D 73 (2006) 126003 [hep-th/0507158] [INSPIRE].

[12] G. Shiu, G. Torroba, B. Underwood and M.R. Douglas, Dynamics of warped flux compactifications, JHEP 06 (2008) 024 [arXiv:0803.3068] [INSPIRE].

[13] L. Randall and R. Sundrum, A large mass hierarchy from a small extra dimension, Phys. Rev. Lett. 83 (1999) 3370 [hep-ph/9905221] [INSPIRE].

[14] S. Franco, D. Galloni, A. Retolaza and A. Uranga, On axion monodromy inflation in warped throats, JHEP 02 (2015) 086 [arXiv: 1405.7044] [INSPIRE].

[15] K. Kooner, S. Parameswaran and I. Zavala, Warping the weak gravity conjecture, Phys. Lett. B 759 (2016) 402 [arXiv: 1509.07049] [INSPIRE].

[16] A. Hebecker, J. Moritz, A. Westphal and L.T. Witkowski, Axion monodromy inflation with warped KK-modes, Phys. Lett. B 754 (2016) 328 [arXiv: 1512.04463] [InSPIRE].

[17] M. Cicoli, C.P. Burgess and F. Quevedo, Fibre inflation: observable gravity waves from IIB string compactifications, JCAP 03 (2009) 013 [arXiv:0808.0691] [INSPIRE].

[18] K. Freese, J.A. Frieman and A.V. Olinto, Natural inflation with pseudo-Nambu-Goldstone bosons, Phys. Rev. Lett. 65 (1990) 3233 [INSPIRE].

[19] J.E. Kim, H.P. Nilles and M. Peloso, Completing natural inflation, JCAP 01 (2005) 005 [hep-ph/0409138] [INSPIRE].

[20] S. Dimopoulos, S. Kachru, J. McGreevy and J.G. Wacker, N-flation, JCAP 08 (2008) 003 [hep-th/0507205] [INSPIRE].

[21] N. Kaloper and L. Sorbo, A natural framework for chaotic inflation, Phys. Rev. Lett. 102 (2009) 121301 [arXiv:0811.1989] [INSPIRE].

[22] E. Silverstein and A. Westphal, Monodromy in the CMB: gravity waves and string inflation, Phys. Rev. D 78 (2008) 106003 [arXiv:0803.3085] [INSPIRE].

[23] L. McAllister, E. Silverstein and A. Westphal, Gravity waves and linear inflation from axion monodromy, Phys. Rev. D 82 (2010) 046003 [arXiv: 0808.0706] [INSPIRE].

[24] A. Westphal, String cosmology - Large-field inflation in string theory, Int. J. Mod. Phys. A 30 (2015) 1530024 [arXiv: 1409.5350] [INSPIRE].

[25] R. Blumenhagen, D. Herschmann and E. Plauschinn, The challenge of realizing F-term axion monodromy inflation in string theory, JHEP 01 (2015) 007 [arXiv:1409.7075] [INSPIRE].

[26] A. Hebecker, P. Mangat, F. Rompineve and L.T. Witkowski, Tuning and backreaction in F-term axion monodromy inflation, Nucl. Phys. B 894 (2015) 456 [arXiv:1411.2032] [INSPIRE].

[27] R. Blumenhagen et al., A flux-scaling scenario for high-scale moduli stabilization in string theory, Nucl. Phys. B 897 (2015) 500 [arXiv: 1503.07634] [INSPIRE]. 
[28] T. Rudelius, On the possibility of large axion moduli spaces, JCAP 04 (2015) 049 [arXiv: 1409.5793] [INSPIRE].

[29] T. Rudelius, Constraints on axion inflation from the weak gravity conjecture, JCAP 09 (2015) 020 [arXiv: 1503.00795] [INSPIRE].

[30] M. Montero, A.M. Uranga and I. Valenzuela, Transplanckian axions!?, JHEP 08 (2015) 032 [arXiv: 1503.03886] [INSPIRE].

[31] J. Brown, W. Cottrell, G. Shiu and P. Soler, Fencing in the swampland: quantum gravity constraints on large field inflation, JHEP 10 (2015) 023 [arXiv: 1503. 04783] [INSPIRE].

[32] I. García-Etxebarria, T.W. Grimm and I. Valenzuela, Special points of inflation in flux compactifications, Nucl. Phys. B 899 (2015) 414 [arXiv:1412.5537] [InSPIRE].

[33] T. Kobayashi, A. Oikawa and H. Otsuka, New potentials for string axion inflation, Phys. Rev. D 93 (2016) 083508 [arXiv:1510.08768] [InSPIRE].

[34] N. Cabo Bizet, O. Loaiza-Brito and I. Zavala, Mirror quintic vacua: hierarchies and inflation, arXiv:1605.03974 [INSPIRE].

[35] T.W. Grimm and J. Louis, The effective action of $N=1$ Calabi-Yau orientifolds, Nucl. Phys. B 699 (2004) 387 [hep-th/0403067] [INSPIRE].

[36] P. Candelas and X.C. de la Ossa, Comments on conifolds, Nucl. Phys. B 342 (1990) 246 [INSPIRE].

[37] P. Candelas, X.C. De La Ossa, P.S. Green and L. Parkes, A pair of Calabi-Yau manifolds as an exactly soluble superconformal theory, Nucl. Phys. B 359 (1991) 21 [INSPIRE].

[38] G. Curio, A. Klemm, D. Lüst and S. Theisen, On the vacuum structure of type-II string compactifications on Calabi-Yau spaces with H fluxes, Nucl. Phys. B 609 (2001) 3 [hep-th/0012213] [INSPIRE].

[39] M.-x. Huang, A. Klemm and S. Quackenbush, Topological string theory on compact Calabi-Yau: Modularity and boundary conditions, Lect. Notes Phys. 757 (2009) 45 [hep-th/0612125] [INSPIRE].

[40] R. Blumenhagen, A conic application of the LVS, talk given at the Conference on Aspects of String Phenomenology and Cosmology, May 12-13, ICTP, Trieste, Italy (2016).

[41] A. Hebecker, P. Mangat, F. Rompineve and L.T. Witkowski, Winding out of the swamp: evading the weak gravity conjecture with F-term winding inflation?, Phys. Lett. B $\mathbf{7 4 8}$ (2015) 455 [arXiv: 1503.07912] [INSPIRE].

[42] P. Berglund et al., Periods for Calabi-Yau and Landau-Ginzburg vacua, Nucl. Phys. B 419 (1994) 352 [hep-th/9308005] [INSPIRE].

[43] P. Candelas, X. De La Ossa, A. Font, S.H. Katz and D.R. Morrison, Mirror symmetry for two parameter models. 1., Nucl. Phys. B 416 (1994) 481 [hep-th/9308083] [INSPIRE].

[44] P. Kaste, W. Lerche, C.A. Lütken and J. Walcher, D-branes on K3 fibrations, Nucl. Phys. B 582 (2000) 203 [hep-th/9912147] [INSPIRE].

[45] A. Giryavets, S. Kachru, P.K. Tripathy and S.P. Trivedi, Flux compactifications on Calabi-Yau threefolds, JHEP 04 (2004) 003 [hep-th/0312104] [INSPIRE].

[46] X. Dong, B. Horn, E. Silverstein and A. Westphal, Simple exercises to flatten your potential, Phys. Rev. D 84 (2011) 026011 [arXiv:1011.4521] [InSPIRE]. 